\title{
Complicity: \\ An Introduction and a Welcome
}

BRENT DAVIS, Co-Editor

University of Alberta

RENATA PHELPS, Co-Editor

Southern Cross University

KRISTOPHER WELLS, Book Review Editor

University of Alberta

Each age interprets its universe in terms of what is currently important to it. Ancient animistic people wanting to make sense of the starry sky saw it as a zoo of people and animals - the Hunter, the Swan, the Lion, the Dog. The mechanical age of the eighteenth century bred a mechanistic philosophy; in the clockwork universe, God was the watchmaker who set the wheels spinning and then stood back to watch his creation turn; the newly discovered constellations of the southern hemisphere included the Octant, the Triangle, and the Microscope. Our present Computer Age sees the universe as an ever-changing flow of information, and if we were to discover the stars today our first instinct would be to try to decode their message.

- Jack Cohen \& Ian Stewart ${ }^{1}$ 


\section{What is Complexity?}

At the first Conference on Complexity Science and Educational Research ${ }^{2}$ Ellen Goldberg of the Santa Fe Institute, noted in her keynote that:

... there is no established definition of complexity or complexity science. If you were to ask ten different people wandering the halls of the Santa Fe Institute, you'd get ten different-and likely ten very distinct-responses.

One of the reasons for this situation is that, in contrast to the analytic science of the Enlightenment, the nascent domain of complexity research defines itself more in terms of its objects of study than its modes of investigation. In one popular account of the emergence of the field, M. Mitchell Waldrop ${ }^{3}$ introduces the diverse interests and the diffuse origins of complexity science through a list that includes such disparate events as the collapse of the Soviet Union, trends in a stock market, the rise of life on earth, the evolution of the eye, and the emergence of mind. It should thus be unsurprising that complexity researchers would offer a diversity of characterizations of their umbrella notion of complexity science.

What has come to be called complexity science first arose in the confluence of several fields-including cybernetics, systems theory, artificial intelligence, and nonlinear dynamics - many of which had begun to appear in the physical sciences in the mid-20th century. More recently, complexity theories have come to be taken up in the social sciences in many and various ways ranging from the highly technical, philosophical, narrative and more recently the applied.

Despite the tremendous diversity, however, there are some important qualities that are common to all complex forms. For example, complex phenomena are adaptive- that is, their dynamics are better described in terms of Darwinian evolution than Newtonian mechanics. Further, each involves a certain spontaneous self-organization, in which coherent collective activities or characters arise in the activities of individual agents. In brief, complexity is concerned with non-linear dynamics, emergence and self-organization. It might be defined as a formal attempt to explore how simple and sometimes non-purposive components in a system can self-organize, emerge or evolve into coherent, purposive and complex wholes.

In more provocative terms - and revealing our own educational biases toward the science of complexity-such adaptive, self-organizing phenomena are learning systems. Significantly, while "learning systems" include individuals, there are many other, nested learning phenomena that are of core interest to educators and educational researchers, including classroom collectives, schools, student bodies, communities, societies, and cultures. 


\section{Complexity and Education}

While this brief exploration highlights the relevance of complexity to the study of education, and teaching and learning more specifically, to date educationalists have been somewhat reserved in finding a voice in the complexity community. Aside from the networked gatherings of the Chaos and Complexity Theory Special Interest Group of the American Educational Research Association (AERA), there has been limited avenue for international dialogue. Until this time, no peer-reviewed forum existed for educationalists to explore the application of complexity to theory and practice.

Yet an increasing number of educational researchers and practitioners are becoming aware of the potential of complexity in stimulating new insights and understandings about learning and teaching. Such researchers are applying complexity's principles in diverse ways, from examination of neural processes, brain functioning and the nature of consciousness to more metaphorical explorations of learning. What such writers have in common is an interest to understand learning, teaching and education from nonlinear perspectives, drawing on the emphasis complexity places on variation as a source and outcome of thinking and the important role of interaction, diversity and redundancy on processes of cognition.

It is our pleasure, then, to be writing this editorial for the first issue of the journal Complicity: An International Journal of Complexity and Education. In brief, Complicity is an international, peer-reviewed journal that publishes scholarly and original research linking complexity and related theories to the field of education at all levels. The journal strives to serve as a forum for both theoretical and practical contributions to this comparatively recent field of educational research and to facilitate the exchange of diverse ideas and points of view related to complexity theories in education.

\section{Why Complicity?}

The choice of title for this journal has been made with some significant consideration. Sharing the same etymological roots as complexity (from the Latin com- together + plectere to braid), complicity prompts our attentions, as educators and researchers, to the way we are immediate and significant participants in a range of emergent phenomena, including the identities of our students and the character of formal education.

We were first alerted to the deep intertwinings of complicity and complexity while reading Jack Cohen and Ian Stewart's ${ }^{4}$ The collapse of chaos: discovering simplicity in a complex world. In a moment of clever word play, these authors cross the terms simplicity and complexity to generate simplexity 
and complicity, using this new pair of words to point to what is, and is not, of interest to complexity science.

For Cohen and Stewart, simplexity refers "to the process whereby a system of rules can engender simple features. Simplexity is the emergence of large-scale simplicities as direct consequences of rules" (p. 411). Among the illustrative examples they offer are Newton's laws and formal mathematics, whose "properties are the direct and inescapable consequences of the rules" (p. 412).

A core issue among complexivists, they note, is that simplexities have not only been taken as models, they have been mistaken as the way things really are. In an effort to interrupt this deeply entrenched habit, Cohen and Stewart propose the notion of complicities as a category of phenomena in which "totally different rules converge to produce similar features, and so exhibit the same large-scale structural patterns" (p. 414).

The educational import of Cohen and Stewart's simplexity/ complicity distinction arises in their summary: "Simplexity merely explores a fixed space of the possible. ... Complicity enlarges it."

\section{The place of Complicity in the educational landscape}

This "enlargement of the possible" is what we imagine will be the potential of this new online publication, Complicity. Through this peer-reviewed journal we hope to contribute to an enlarging of the space of the possible around what it means to educate and to be educated.

To this end, we acknowledge that the tools and expectations of traditional academic discourse might have to be challenged on occasion. A prominent theme across the current complexity science literatures is that the linear narrative and the Euclidean image are inadequate to depict the emergence and the behavior of a complex form. Rather, instances of complex emergence call for webbed, multithreaded tales and nested, scale independent geometries to accommodate forms that can become more intricate, more dense, more pregnant with possibilities.

As Complicity develops we hope to develop a vibrant international network of readers and publishers who will make play with the "possible". Consistent with complexity we hope to foster this learning community through the "webbed, multithreaded tales" enabled through the Internet. The Complexity and Education Website (http:// www.complexityandeducation.ca) provides an opportunity for researchers, educators, graduate students, and pre-service and practicing teachers to explore how complexity influences their research, teaching and learning engagements. This website features information on the annual International Complexity \& Education Confer- 
ence, including conference proceedings, a discussion forum, an online glossary and annotated bibliography, publications, presentations, and other events and announcements.

\section{Our Inaugural Papers}

It is thus our great pleasure to introduce and present the first 5 articles in this first edition of Complicity.

We open with a cautionary tale, offered by Anne Phelan. This invited article is based on a talk that Anne presented as an appointed raconteur at the most recent Complexity Science and Educational Research Conference (Chaffey's Locks, Ontario, 2004). While maintaining a cautious optimism, she reminds us to be attentive to the partialities of any interpretive frame and urges us not to fall into the same sort of uncritical confidence that analytic science has inspired.

Rena Uptis is a seasoned researcher and writer in complexity and education. As an educator with a deep interest in the arts Rena brings a unique and creative perspective to her application of complexity to teaching and learning. Rena's paper provides an exploration of the role of architectural design of educational environments on learning and teaching, particularly in disciplines such as music, drama, dance, and the visual arts. Drawing on ideas of "learning collectives", Rena explores how Froebel kindergartens, Reggio Emilia schools, and Waldorf schools might provide enriched understandings of physical learning environments, drawing implications for school design based on the principles of complexity.

Margaret Sinclair explores the application of complexity in a technology-supported environment. Drawing on the idea of the classroom as an adaptive and self-organizing complex system Sinclair describes three labbased learning contexts. Through this discussion she provides insights into five aspects of complex systems: internal diversity, redundancy, decentralized control, organized randomness and neighbour interactions. Sinclair's paper has both theoretical and practical relevance for educators, highlighting the benefit of teaching and learning structures that allow for sharing, play and individual choice in lab-based technological environments.

Charles Nelson's contribution applies complexity to the area of second language learning, and in particular to composition. Nelson proposes that complexity can assist in understanding how second language learners generate creativity in novel situations, and how building blocks are reproduced, recombined through cross-over and replaced. Nelson's conclusions provide a challenge for educators to consider how teaching structures can support "intertwining rhetoric" into other disciplines and student interests. 
Lynn Fels provides a thought-provoking and creative exploration of 'performance' and 'performative spaces' which challenges teacher educators to reconceptualize what it means to be and become a teacher. "Becoming a teacher is realized within messy, chaotic, generative spaces that we call learning, as emergent possible worlds-as-yet-unlived unfold". These spaces, Fels proposes, become "possible landscapes" for teacher educators. In a rich, poetic piece, Fels challenges us to "interrupt, startle (and) reimagine the classroom habits, engagements, expectations and practices" in order to "help student teachers come to recognize the complexities and complicity inherent within pedagogical spaces".

\section{Book Reviews}

In addition to the aforementioned papers, we are pleased to include a series of critical book reviews in each edition of Complicity. These reviews are carefully crafted to examine foundational and contemporary texts that are coimplicated in the emerging field of complexity and education. In this inaugural issue we present five diverse reviews from authors in Canada, the United States, and Australia.

Marina Basu invites us to revisit Varela, Thompson, and Rosch's influential text The Embodied Mind: Cognitive Science and Human Experience. In her review, Basu draws our attention to the ways in which the authors skillfully weave together Eastern philosophy and Western thought to enhance our understandings of complexity and emergence.

Susan Bowsfield engages us with Deacon's influential text, The Symbolic Species: The Co-Evolution of Language and the Brain. In this review, Bowsfield outlines key elements of Deacon's co-evolutionary theory of homo sapien development and poses critical questions for educators who are interested in exploring and prompting complexivist sensibilities in their classrooms.

S. Megan Che contributes a skillful review of the lengthy edited compilation Animal Social Complexity: Intelligence, Culture, and Individualized Societies. Che's descriptive review highlights how De Waal \& Tyack's text provides impetus for readers to extend their own understandings of complexity beyond the human world to investigate the dynamical relationships between human and animal social complexity, cognition, and intelligence.

Tara J. Fenwick provides readers with a critical and cautionary analysis of Cutright's edited text Chaos Theory and Higher Education: Leadership, Planning and Policy. Fenwick lays challenge to the trend of generic applications of chaos / complexity as she calls for a more sophisticated theoretical analysis that moves beyond a complicated recipe style format to a more complex 
interrogation that brings into question issues of power, politics, subjectivities, and self-reflexivity.

Warren Sellers provocatively challenges and disrupts traditional conventions by providing both a written and visual reading of Merrell's text Simplicity and Complexity: Pondering Literature, Science and Painting. In this montage, Sellers co-implicates his reading not in the promise of a tidy summation of Merrell's text, rather he seeks to engage the paradoxes, tensions, interconnections, and interrelationships as generative grounds from which to explore creative and innovative educational possibilities.

Individually and collectively these reviews develop multiple narrative tellings and (re)tellings that provide for a rich and diverse collection of reviews that engage us in a thoughtful dialogue variously composed of part appraisal, critique, and caution.

\section{Welcome}

As co-editors of this new journal, Complicity, we are excited about the possibilities that this publication will open up for shared dialogue. We look forward to joining with you in creating a vibrant and challenging conversation about what complexity means to us as an educational community. We encourage new readers and writers to actively participate in order for us to build a collective international voice in this emerging field.

\section{Endnotes}

1. Cohen, J.S. \& I. Stewart. Collapse of Chaos: Discovering Simplicity in a Complex World. London: Penguin, 1995, p. 288.

2. The first Conference on Complexity Science and Educational Research was held in Edmonton, Alberta in October, 2003. Details on the conference and its Proceedings are available at http:/ / www.complexityandeducation.ca under the "Conferences" link.

3. Waldrop, M. Complexity: The Emerging Science at the Edge of Order and Chaos. London: Penguin, 1992.

4. Cohen \& Stewart, Collapse of Chaos. 